\title{
Locally asymptotically rank-based procedures for testing autoregressive moving average dependence
}

\author{
(linear serial rank statistics/quadratic rank statistics/rank portmanteau statistics/asymptotic sufficiency/asymptotically maximin most \\ powerful tests)
}

\author{
Marc Hallin ${ }^{\dagger}$ and Madan L. Puri ${ }^{\ddagger}$

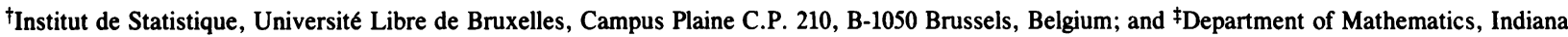 \\ University, Bloomington, IN 47405
}

Communicated by Herbert Robbins, November 24, 1987

\begin{abstract}
The problem of testing a given autoregressive moving average (ARMA) model (in which the density of the generating white noise is unspecified) against other ARMA models is considered. A distribution-free asymptotically most powerful test, based on a generalized linear serial rank statistic, is provided against contiguous ARMA alternatives with specified coefficients. In the case in which the ARMA model in the alternative has unspecified coefficients, the asymptotic sufficiency (in the sense of Hajek) of a finite-dimensional vector of rank statistics is established. This asymptotic sufficiency is used to derive an asymptotically maximin most powerful test, based on a generalized quadratic serial rank statistic. The asymptotically maximin optimal test statistic can be interpreted as a rank-based, weighted version of the classical Box-Pierce portmanteau statistic, to which it reduces, in some particular problems, under gaussian assumptions.
\end{abstract}

\section{Section 1. Rank Tests for Time Series Analysis}

Time series analysis is one of the areas in statistics whose development has been most impressive during the past two decades, and its importance for practical applications, ranging from economics to engineering, has been widely recognized. However, in spite of the growing interest in the subject, and in spite of the fact that the need for robust or rankbased procedures has been emphasized by many authors, little has been done to introduce such procedures (especially rank-based ones) in time series context.

A step toward a systematic treatment of this subject was taken in three papers by Hallin et al. $(1,2,8)$, in which asymptotically optimal and maximin-optimal rank tests (in the Pitman-Noether sense) were derived for the problem of testing randomness against alternatives of autoregressive moving average (ARMA) dependence. Two classes of serial rank statistics were introduced for that purpose.

The first one is the class of linear serial rank statistics, of order $p$, of the form

$$
S^{(n)}=(n-p)^{-1} \sum_{t=p+1}^{n} a^{(n)}\left(R_{t}^{(n)}, R_{t-1}^{(n)}, \ldots, R_{t-p}^{(n)}\right)
$$

where $R_{t}^{(n)}$ denotes the rank of $X_{t}^{(n)}$ in some observed series $\mathbf{X}^{(n)}=\left(X_{1}^{(n)}, \ldots, X_{n}^{(n)}\right)$ of length $n$, and $a^{(n)}(\ldots)$ is some score function. Of special interest is the particular linear serial rank statistic-i.e., the rank autocorrelation $r_{i ; f}^{(n)}$ (for definition, see Eq. 9) of order $i(=1, \ldots, n-2)$ associated with density $f(\cdot)$ that enjoys (cf. ref. 2 ) most of the asymptotic properties of the usual sample autocorrelations $r_{i}^{(n)}$ for gaussian series.

The publication costs of this article were defrayed in part by page charge payment. This article must therefore be hereby marked "advertisement" in accordance with 18 U.S.C. $\$ 1734$ solely to indicate this fact.
The second class of rank statistics considered (in ref. 2) is that of quadratic serial rank statistics, which are quadratic forms of linear serial rank statistics. Of particular interest are the rank portmanteau statistics $\sum_{i=1}^{\kappa}\left(r_{i ; f}^{(n)}\right)^{2}$ that provide a rank version of the classical portmanteau statistics $\Sigma_{i=1}^{\kappa}\left(r_{i}^{(n)}\right)^{2}$ of Box and Pierce (3).

Section 2. Testing an ARMA Model Against Another ARMA Model: Asymptotically Most Powerful Test

The problem of testing a given ARMA model (with unspecified density function) against other ARMA models is important because of its implications in the various identification and validation steps that are part of any time series modelbuilding procedure.

Denote by $H^{(n)}(\mathrm{A}, \mathrm{B} ; \cdot)$ the null hypothesis under which the observed series $\mathbf{X}^{(n)}$ is generated by the ARMA $\left(p_{1}, q_{1}\right)$ model

$$
\begin{aligned}
A(L) X_{t} & =X_{t}-A_{1} X_{t-1}-\ldots-A_{p_{1}} X_{t-p_{1}} \\
& =\varepsilon_{t}+B_{1} \varepsilon_{t-1}+\ldots+B_{q_{1}} \varepsilon_{t-q_{1}} \\
& =B(L) \varepsilon_{t}, \quad t \in \mathbb{Z},
\end{aligned}
$$

where $\left\{\varepsilon_{t}\right\}$ is an independent white-noise process with unspecified density function $f[L$ denotes the lag operator, and $A(L)=1-A_{1} L-\ldots-A_{p_{1}} L^{p_{1}}, B(L)=1+B_{1} L+\ldots+$ $B_{q_{1}} L^{q_{1}}$ are difference operators].

We assume the following conditions.

(i) $A(L)$ and $B(L)$ are of orders $p_{1}$ and $q_{1}$, respectively (i.e., $\left.A_{p_{1}} \neq 0 \neq B_{q_{1}}\right)$.

(ii) The polynomials $A(z)$ and $B(z), z \in \mathbb{C}$ have no common roots.

(iii) The polynomials $A(z)$ and $B(z), z \in \mathbb{\&}$ have all their roots outside the unit circle.

(iv) $\varepsilon_{t}$ has finite moments up to the sixth order. $E\left(\varepsilon_{t}\right)=0$ and $E\left(\varepsilon_{t}^{2}\right)=\sigma^{2}$ (unspecified).

(v) $f(x)$ is (a.e.) derivable [denote by $f^{\prime}(x)$ its derivative] and absolutely continuous on finite intervals and has finite Fisher's information $I(f): 0<I(f)=E\left[\left(f^{\prime}\left(\varepsilon_{t}\right) / f\left(\varepsilon_{t}\right)\right)^{2}\right]<\infty$.

(vi) Denote by $F(x)$ the cumulative distribution function associated with $f(x)$, and let $F^{-1}(u)=\inf \{x \mid F(x) \geq u\}, 0<u$ $<1$. Define $\phi(x)$ as $-f^{\prime}\left(F^{-1}(F(x))\right) / f\left(F^{-1}(F(x))\right)$. Assume that $\phi(x)$ is a.e. derivable and that its derivative $\phi^{\prime}(x)$ is (a.e.) lipschitzian and square integrable: $\left|\phi^{\prime}(x)-\phi^{\prime}(y)\right|<K|x-y|$, and $\int_{0}^{1} \phi^{\prime 2}\left(F^{-1}(u)\right) d u<\infty$. Note that $\phi(x)$ is a.e. equal to $-f^{\prime}(x) / f(x)$.

Conditions $i v-v i$ are assumed to hold throughout this paper. Some results (mainly those involving $f$-rank autocorrelations) require an additional assumption on $f$, as follows:

(vii) $f$ is strongly unimodal [cf. page 15 of Hájek and Šidák (4)].

Abbreviation: ARMA, autoregressive moving average. 
The alternative hypotheses of interest here are those under which $\mathbf{X}^{(n)}$ is an observed series from some other ARMA model $\alpha(L) X_{t}=\beta(L) \varepsilon_{t}$ distinct from Eq. 2. To investigate locally optimal procedures, we shall consider sequences of alternatives that are contiguous to the null hypotheses. Let

$$
\begin{aligned}
\alpha^{(n)}(L) X_{t} & =X_{t}-\alpha_{1}^{(n)} X_{t-1}-\ldots-\alpha_{p_{2}}^{(n)} X_{t-p_{2}} \\
& =\varepsilon_{t}+\beta_{1}^{(n)} \varepsilon_{t-1}+\ldots+\beta_{q_{2}}^{(n)} \varepsilon_{t-q_{2}} \\
& =\beta^{(n)}(L) \varepsilon_{t}
\end{aligned}
$$

be a sequence of $\operatorname{ARMA}\left(p_{2}, q_{2}\right)$ models, with $p_{2} \geq p_{1}, q_{2} \geq$ $q_{1}, \alpha_{p_{2}} \neq 0 \neq \beta_{q_{2}}$, and

$$
\begin{aligned}
& \alpha_{i}^{(n)}=\left\{\begin{array}{rl}
A_{i}+n^{-1 / 2} \gamma_{i}, & 1 \leq i \leq p_{1} \\
n^{-1 / 2} \gamma_{i}, & p_{1}<i \leq p_{2}
\end{array},\right. \\
& \beta_{i}^{(n)}=\left\{\begin{array}{rr}
B_{i}+n^{-1 / 2} \delta_{i}, & 1 \leq i \leq q_{1} \\
n^{-1 / 2} \delta_{i}, & q_{1}<i \leq q_{2}
\end{array} .\right.
\end{aligned}
$$

Furthermore, assume that the polynomials $\alpha^{(n)}(z)$ and $\beta^{(n)}(z)$, $z \in \mathbb{C}$ have no root that is common for any value of $n \in \mathbb{N}$. Denote by $\boldsymbol{\gamma}$ and $\delta$ the vectors $\left(\gamma_{1}, \ldots, \gamma_{p_{2}}\right)$ and $\left(\delta_{1}, \ldots\right.$, $\left.\delta_{q_{2}}\right)$, respectively, and by $K^{(n)}(A, B ; \gamma, \delta ; f)$ the sequence of ARMA $\left(p_{2}, q_{2}\right)$ alternatives (Eq. 3) corresponding to specified $\boldsymbol{\gamma}, \delta$, and $f$.

Denoting by $\left\{Z_{t}\right\}$ the filtered process $(A(L) / B(L)) X_{t}$ (i.e., the exact residuals with respect to Eq. 2), we note that, under the null hypothesis, $\left\{Z_{t}\right\}$ is a white-noise process and, under the alternative hypothesis, $K^{(n)}(\mathbf{A}, \mathbf{B} ; \boldsymbol{\gamma}, \boldsymbol{\delta} ; f)$ (see Eqs. 3 and 4$),\left\{Z_{t}\right\}$ is generated by a general linear model of the form

$$
Z_{t}-n^{-1 / 2} \sum_{i=1}^{\infty} a_{i} Z_{t-i}=\varepsilon_{t}+n^{-1 / 2} \sum_{i=1}^{\infty} b_{i} \varepsilon_{t-i}, \quad t \in \mathbb{Z},
$$

where

$$
\begin{aligned}
& a_{i}=\sum_{j=1}^{\min \left(p_{2}, i+p_{1}-1\right)} \gamma_{j} G_{i-j} \quad \text { and } \\
& b_{i}=\sum_{j=1}^{\min \left(q_{2}, i+q_{1}-1\right)} \delta_{j} H_{i-j}, \quad i=1,2, \ldots .
\end{aligned}
$$

Here $G_{u}$ and $H_{u}, u \in \mathbb{Z}$ are the Green's functions of the operators $A(L)$ and $B(L)$, respectively, $\left(p_{1}>0\right.$ and $\left.q_{1}>0\right)$ :

$$
\begin{aligned}
& {[A(L)]^{-1}=1+\sum_{u=1}^{\infty} G_{u} L^{u} \quad \text { and }} \\
& {[B(L)]^{-1}=1+\sum_{u=1}^{\infty} H_{u} \mathrm{~L}^{u},}
\end{aligned}
$$

with

$$
\sum_{u=1}^{\infty}\left|G_{u}\right|<\infty \quad \text { and } \quad \sum_{u=1}^{\infty}\left|H_{u}\right|<\infty
$$

which imply $\Sigma_{i=1}^{\infty}\left|a_{i}\right|<\infty$ and $\Sigma_{i=1}^{\infty}\left|b_{i}\right|<\infty$.

Let $R_{t}^{(n)}$ be the rank of $Z_{t}$ among $\left(Z_{1}, \ldots, Z_{n}\right)$, and define the rank autocorrelation coefficient associated with density $f$ (or $f$-rank autocorrelation) of order $i$ as

$$
\begin{aligned}
r_{i ; f}^{(n)}= & {\left[(n-i)^{-1} \sum_{t=i+1}^{n} \phi\left(F^{-1}\left(\frac{R_{t}^{(n)}}{n+1}\right)\right)\right.} \\
& \left.\times F^{-1}\left(\frac{R_{t-i}^{(n)}}{n+1}\right)-m^{(n)}\right] / \sigma^{(n)},
\end{aligned}
$$

where

$$
m^{(n)}=(n(n-1))^{-1} \sum_{1 \leq i_{1} \neq i_{2} \leq n} \phi\left(F^{-1}\left(\frac{i_{1}}{n+1}\right)\right) F^{-1}\left(\frac{i_{2}}{n+1}\right)[10]
$$

and

$$
\begin{gathered}
{\left[\sigma^{(n)}\right]^{2}=\frac{1}{n(n-1)} \sum_{1 \leq i_{1} \neq i_{2} \leq n}\left[\phi\left(F^{-1}\left(\frac{i_{1}}{n+1}\right)\right) F^{-1}\left(\frac{i_{2}}{n+1}\right)\right]^{2}} \\
+2 \frac{(n-2 i)}{(n-i)} \cdot \frac{1}{n(n-1)(n-2)} \sum_{i \leq i_{1} \neq i_{2} \neq i_{3} \leq n}\left[\phi\left(F^{-1}\left(\frac{i_{1}}{n+1}\right)\right)\right. \\
\left.\phi\left(F^{-1}\left(\frac{i_{2}}{n+1}\right)\right) F^{-1}\left(\frac{i_{2}}{n+1}\right) F^{-1}\left(\frac{i_{3}}{n+1}\right)\right] \\
+\frac{n^{2}-n(2 i+3)+i^{2}+5 i}{n(n-1)(n-2)(n-3)(n-i)} \sum_{1 \leq i_{1} \neq i_{2} \neq i_{3} \neq \sum_{4} \leq n} \sum_{\phi} \\
\left.\left[F^{-1}\left(\frac{i_{1}}{n+1}\right)\right) \phi\left(F^{-1}\left(\frac{i_{2}}{n+1}\right)\right) F^{-1}\left(\frac{i_{3}}{n+1}\right) F^{-1}\left(\frac{i_{4}}{n+1}\right)\right]
\end{gathered}
$$

Then

Proposition 2.1. The asymptotically most powerful test for $\mathrm{H}^{(\mathrm{n})}(\mathrm{A}, \mathrm{B} ; \cdot)$ against $\mathrm{K}^{(\mathrm{n})}(\mathrm{A}, \mathrm{B} ; \boldsymbol{\gamma}, \boldsymbol{\delta} ; \mathrm{f})$ at given level $\alpha$ rejects if

$$
n^{1 / 2} \sum_{i=1}^{n-2}\left(a_{i}+b_{i}\right) r_{i ; f}^{(n)}>\left[\sum_{i=1}^{n-2}\left(a_{i}+b_{i}\right)^{2}\right]^{1 / 2} k_{1-\alpha}
$$

where $\mathrm{k}_{1-\alpha}$ denotes the $(1-\alpha)$-quantile of the standard normal distribution.

The proof of this Proposition is given in ref. 5.

\section{Section 3. Asymptotic Sufficiency of $\boldsymbol{f}$-Rank Autocorrelations}

Consider the general problem of testing $H^{(n)}$ against $K^{(n)}$ where $\boldsymbol{H}^{(n)}=\left\{l_{0}^{(n)}\right\}$ and $K^{(n)}=\left\{l_{1}^{(n)}\right\}$ are families of densities for some observed $X^{(n)}$ defined over a sample space $\left(\mathscr{L}^{(n)}\right.$, $\left.\mathscr{A}^{(n)}\right)$. Recall that the envelope power function $\beta\left(\alpha, H^{(n)}\right.$, $K^{(n)}$ ) for this problem is defined as

$$
\beta\left(\alpha, H^{(n)}, K^{(n)}\right)=\sup _{\psi^{(n)}} \inf _{l_{1}^{(n)} \in K^{(n)}} E_{l_{1}^{(n)}}\left[\psi^{(n)}\right]
$$

where the supremum is taken over all the tests $\psi^{(n)}$ satisfying, for fixed $\alpha \in[0,1]$,

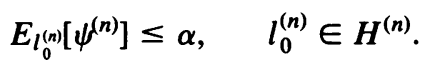

Let $T^{(n)}$ be some statistic from $\left(\mathscr{X}^{(n)}, \mathscr{A}^{(n)}\right)$ to some range space $\left(\mathscr{T}^{(n)}, \mathscr{B}^{(n)}\right)$. Define the envelope power function $\beta_{T}(\alpha$, $H^{(n)}, K^{(n)}$ ) associated with $T^{(n)}$ by taking the supremum, in Eq. 12, over the set of all $T^{(n)}$-measurable tests $\psi^{(n)}$ satisfying Eq. 13. If we denote by $H_{T}^{(n)}$ and $K_{T}^{(n)}$ the families of densities [on $\left(\mathscr{T}^{(n)}, \mathscr{B}^{(n)}\right)$ ] induced from $H^{(n)}$ and $K^{(n)}$ by $T^{(n)}$, then clearly

$$
\beta_{T}\left(\alpha, H^{(n)}, K^{(n)}\right)=\beta\left(\alpha, H_{T}^{(n)}, K_{T}^{(n)}\right) .
$$

Obviously, we also have

$$
\beta\left(\alpha, H^{(n)}, K^{(n)}\right) \geq \beta_{T}\left(\alpha, H^{(n)}, K^{(n)}\right) .
$$

If, however, an equality in Eq. 15 is achieved for every $\alpha \in$ $[0,1]$ [this happens, e.g., if $T^{(n)}$ is sufficient for the structure 
$\left.\left(\mathscr{C}^{(n)}, \mathbb{A}^{(n)}, H^{(n)} \cup K^{(n)}\right)\right]$, then $T^{(n)}$ can be considered, in some sense, as sufficient for the problem of testing $H^{(n)}$ against $K^{(n)}$.

Accordingly [see LeCam (6) and theorem VII of Hájek and Sidák (4)] we say that $T^{(n)}$ is asymptotically sufficient for the problem of testing $H^{(n)}$ against $K^{(n)}$ if

$\lim _{n \rightarrow \infty}\left[\beta\left(\alpha, H^{(n)}, K^{(n)}\right)-\beta_{T}\left(\alpha, H^{(n)}, K^{(n)}\right)\right]=0$,

$$
\alpha \in[0,1]
$$

An important consequence is that if $T^{(n)}$ is asymptotically sufficient for $H^{(n)}$ against $K^{(n)}$, then any asymptotically maximin most powerful test $\psi^{(n) *}$ for $H_{T}^{(n)}$ against $K_{T}^{(n)}$ is also asymptotically maximin most powerful for $H^{(n)}$ against $K^{(n)}$.

Let us consider now the problem of testing $H^{(n)}(\mathrm{A}, \mathrm{B} ; \cdot)$ against unspecified ARMA alternatives. Denote by

$$
\begin{aligned}
K^{(n)}\left(\mathbf{A}, \mathbf{B} ; \mathbb{R}^{p_{2}}, \mathbb{R}^{q_{2}} ; f\right) \\
=U\left\{K^{(n)}(\mathbf{A}, \mathbf{B} ; \boldsymbol{\gamma}, \boldsymbol{\delta} ; f) \mid \gamma \in \mathbb{R}^{p_{2}}, \boldsymbol{\delta} \in \mathbb{R}^{q_{2}}\right\},
\end{aligned}
$$

the alternative under which the observed series $\mathbf{X}^{(n)}$ is generated by some ARMA model (Eqs. 3 and 4, where $\gamma$ and $\delta$ take on unspecified values, although being of specified dimensions $p_{2}$ and $q_{2}$. Let also $d \in \mathbb{R}^{+}$, and denote by $\mathscr{D}(d)$ the set of values of $(\gamma, \delta) \in \mathbb{R}^{p_{2}} \times \mathbb{R}^{q_{2}}$ such that $\|\mathbf{a}+\mathbf{b}\| \geq d$, with $\mathbf{a}$ and $\mathbf{b}$ given in Eq. 6. In order to obtain asymptotically maximin most powerful tests, we need to consider subhypotheses of the form

$$
\begin{aligned}
K^{(n)}\left(\mathbf{A}, \mathbf{B} ; \mathbb{R}^{p_{2}},\right. & \left.\mathbb{R}^{q_{2}} ; f \mid d\right) \\
& =U\left\{K^{(n)}(\mathbf{A}, \mathbf{B} ; \boldsymbol{\gamma}, \boldsymbol{\delta} ; f) \mid(\boldsymbol{\gamma}, \boldsymbol{\delta}) \in \mathscr{D}(d)\right\} .
\end{aligned}
$$

We are now able to characterize a finite-dimensional rankbased statistic that is asymptotically sufficient (in the sense defined above) for testing a given ARMA model against another, unspecified, one.

Proposition 3.1. Let $\left\{\psi_{\mathrm{t}}^{(1)}, \ldots, \psi^{\left(\mathrm{p}_{1}+\mathrm{q}_{1}\right)}\right\}$ denote an arbitrary fundamental system of solutions of the homogeneous equation

$$
\mathrm{A}(\mathrm{L}) \mathrm{B}(\mathrm{L}) \psi_{\mathrm{t}}=0, \quad \mathrm{t} \in \mathbb{Z} \text {. }
$$

Writing $\pi$ for $\max \left(\mathrm{p}_{2}-\mathrm{p}_{1}, \mathrm{q}_{2}-\mathrm{q}_{1}\right)$, consider the $\max \left(\mathrm{p}_{1}+\right.$ $\left.\mathrm{q}_{2}, \mathrm{p}_{2}+\mathrm{q}_{1}\right)$-tuple of rank statistics [assume $\mathrm{n} \geq \max \left(\mathrm{p}_{2}-\mathrm{p}_{1}\right.$ $\left.\left.+3, \mathrm{q}_{2}-\mathrm{q}_{1}+3, \mathrm{p}_{1}+\mathrm{q}_{1}+2\right)\right]$

$$
\begin{aligned}
\mathbf{T}_{\psi ; \mathrm{f}}^{(\mathrm{n})}=\left(\mathrm{r}_{\mathrm{i} ; \mathrm{f}}^{(\mathrm{n})}, \ldots, \mathrm{r}_{\pi ; \mathrm{f}}^{(\mathrm{n})}, \sum_{\mathrm{i}=\pi+1}^{\mathrm{n}-2} \psi_{\mathrm{i}}^{(1)} \mathbf{r}_{\mathrm{i} ; \mathrm{f}}^{(\mathrm{n})}, \ldots,\right. \\
\left.\sum_{\mathrm{i}=\pi+1}^{\mathrm{n}-2} \psi_{\mathrm{i}}^{\left(\mathrm{p}_{1}+\mathrm{q}_{1}\right)} r_{\mathrm{i} ; \mathrm{f}}^{(\mathrm{n})}\right)^{\prime} .
\end{aligned}
$$

Then, for any $\mathrm{d} \in \mathbb{R}^{+}, \mathrm{T}_{\psi ; \mathrm{f}}^{(\mathrm{n})}$ is asymptotically sufficient for the problem of testing $\mathrm{H}^{(\mathrm{n})}(\mathrm{A}, \mathrm{B} ; \cdot)$ against Eq. 18.

The proof mainly consists in establishing that the sufficient conditions for asymptotic sufficiency of theorem VII 1.1 of Hájek and Šidák (4) are satisfied. Let $l_{0 ; f}^{(n)}\left(\mathbf{X}^{(n)}\right)=$ $\Pi_{t=1}^{n} f\left(Z_{t}^{(n)}\right)$. Then we have to show that there exist $\mathbf{T}_{\psi ; f^{-}}^{(n)}$ measurable variables $h_{\gamma, \delta}^{(n)}$, such that the functions

$$
\bar{l}_{\gamma, \delta}^{(n)}=l_{0 ; f}^{(n)} h_{\gamma, \delta}^{(n)}
$$

are densities for all $(\boldsymbol{\gamma}, \delta) \in \mathscr{D}(d)$ and $n$ ( $n$ sufficiently large) and satisfy

$$
\lim _{n \rightarrow \infty} \sup _{\mathscr{D}(\mathrm{d})}\left\|l_{\gamma, \delta}^{(n)}-\bar{l}_{\gamma, \delta}^{(n)}\right\|_{L_{1}}=0,
$$

where $\|p-q\|_{L_{1}}$ is the $L_{1}$-distance $\int|p-q| d \mu$ for densities $p$ and $q$ defined with respect to the $\sigma$-finite measure $\mu$ [for details, see Hallin and Puri (5)].

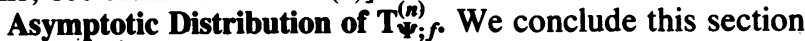
by establishing the joint asymptotic normality of any asymptotically sufficient vector $\mathbf{T}_{\Psi ; f}^{(n)}$

Proposition 3.2. $\mathrm{n}^{1 / 2} \mathbf{T}_{\Psi ; \mathrm{f}}^{(\mathrm{n})}$ is asymptotically normal, with mean 0 under $\mathrm{H}^{(\mathrm{n})}(\mathbf{A}, \mathbf{B} ; \cdot)$ mean $\left(\left(\mathrm{a}_{1}+\mathrm{b}_{1}\right), \ldots,\left(\mathrm{a}_{\pi}+\mathrm{b}_{\pi}\right)\right.$ $\left.\Sigma_{i=\pi+1}^{\infty} \psi_{i}^{(1)}\left(a_{i}+b_{i}\right), \ldots, \Sigma_{i=\pi+1}^{\infty} \psi_{i}^{\left(p_{1}+q_{1}\right)}\left(a_{i}+b_{i}\right)\right)\left[\sigma^{2}(I(f)]^{1 / 2}\right.$ under $\mathrm{K}^{(\mathrm{n})}(\mathbf{A}, \mathbf{B} ; \boldsymbol{\gamma}, \boldsymbol{\delta} ; \mathbf{f})$, and full-rank covariance matrix

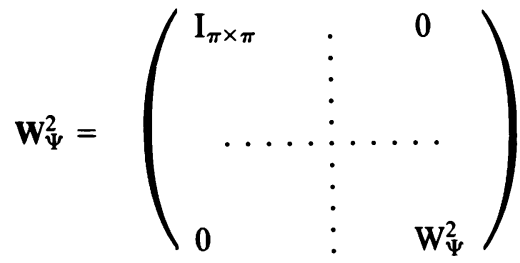

under both $\mathrm{H}^{(\mathrm{n})}(\mathbf{A}, \mathbf{B} ; \cdot)$ and $\mathrm{K}^{(\mathrm{n})}(\mathbf{A}, \mathbf{B} ; \boldsymbol{\gamma}, \boldsymbol{\delta} ; \mathrm{f})$, where $\mathrm{W}_{\Psi}^{2}=$ $\left(\mathrm{W}_{\psi ; \mathbf{k} \mathrm{l}}\right)$ and $\mathrm{W}_{\psi ; \mathrm{kl}}=\Sigma_{\mathrm{i}=\pi+1}^{\infty} \psi_{\mathrm{i}}^{(\mathrm{k})} \psi_{\mathrm{i}}^{(\mathrm{l})}<\infty ; \mathrm{k}, \mathrm{l}=1, \ldots, \mathrm{p}_{1}+$ $\mathrm{q}_{1}$.

Proof: Let $\kappa>\pi$, and consider an arbitrary linear combination of $\log L^{(n)}\left(Z^{(n)}\right), n^{1 / 2} r_{1: f}^{(n)} \ldots, n^{1 / 2} r_{\pi ; f}^{(n)}, n^{1 / 2} \sum_{i=\pi+1}^{k}$ $\psi_{i}^{(1)} r_{i ; f}^{(n)} \ldots$ and $n^{1 / 2} \Sigma_{i=\pi+1}^{\kappa} \psi_{i}^{\left(p_{1}+q_{1}\right)} r_{i ; f}^{(n)}$. [Here $\log L^{(n)}\left(\mathbf{Z}^{(n)}\right)$ denotes the $\log$ likelihood ratio for testing $H^{(n)}(\mathbf{A}, \mathbf{B} ; f)$ against $\left.K^{(n)}(\mathbf{A}, \mathbf{B} ; \boldsymbol{\gamma}, \delta ; f)\right]$. The asymptotic normality under the null hypothesis of this linear combination can easily be established along the lines of proposition 3.1 of Hallin et al. (2), and then using theorem 7.7.1 of Anderson (7), we obtain the asymptotic normality of this linear combination in the case when $\kappa=\infty$. Proposition 3.2 then follows from LeCam's third lemma (6).

The nonsingularity of $W_{\Psi}^{2}$, hence that of $W_{\Psi}^{2}$, results from the fact that the $\Psi_{t}^{(j)}$ values are linearly independent solutions of Eq. 19.

Section 4. Testing an ARMA Model Against Another ARMA Model: Asymptotically Maximin Most Powerful Tests

Generalized Quadratic Serial Rank Statistics. Quadratic serial rank statistics, providing rank-based versions of the portmanteau statistic of Box and Pierce (3) were introduced in Hallin et al. $(2,8)$ to palliate the poor overall performances of linear serial rank statistics in the problem of testing randomness against unspecified ARMA alternatives. The asymptotic (central and noncentral) $\chi^{2}$ distributions of such quadratic statistics (when computed from the ranks of the filtered process $\left.\left\{Z_{t}\right\}\right)$ can easily be obtained under $H^{(n)}(\mathbf{A}$, $\mathbf{B} ; \cdot)$ and $K^{(n)}(\mathbf{A}, \mathbf{B} ; \boldsymbol{\gamma}, \boldsymbol{\delta} ; f)$.

Since, however, the asymptotically most powerful test for $H^{(n)}(\mathbf{A}, \mathbf{B} ; \cdot)$ against $K^{(n)}(\mathbf{A}, \mathbf{B} ; \boldsymbol{\gamma}, \delta ; f)$ cannot, in general, be found within the class of linear serial rank tests, quadratic serial rank statistics cannot be expected to provide optimal tests against unspecified values of $\boldsymbol{\gamma}$ and $\boldsymbol{\delta}$ (unless, e.g., $p_{1}=$ $0=q_{1}$ ). Consequently a more general type of quadratic rank statistics has to be considered. To this end, let $\mathbf{S}^{(n)}-\mathbf{m}^{(n)}=$ $\left(S_{1}^{(n)}-m_{1}^{(n)}, \ldots, S_{l}^{(n)}-m_{l}^{(n)}\right)^{\prime}$ be a column vector of generalized linear serial rank statistics centered about their means [under $H^{(n)}(\mathbf{A}, \mathbf{B} ; \cdot)$ ]. Assuming that $n^{1 / 2}\left(\mathbf{S}^{(n)}-\mathbf{m}^{(n)}\right)$ has an asymptotic covariance matrix $\mathbf{V}^{2}$ of full rank, we define a generalized quadratic serial rank statistic as a statistic of the form $Q^{(n)}=n\left(\mathbf{S}^{(n)}-\mathbf{m}^{(n)}\right)^{\prime} \mathbf{V}^{-2}\left(\mathbf{S}^{(n)}-\mathbf{m}^{(n)}\right)$

Since we have shown that asymptotically sufficient statistics $\mathbf{T}_{\Psi ; f}^{(n)}$ exist for testing $H^{(n)}(\mathbf{A}, \mathbf{B} ; \cdot)$ against $K^{(n)}(\mathbf{A}, \mathbf{B} ; \boldsymbol{\gamma}, \boldsymbol{\delta}$; $f)$, it is intuitively quite natural to consider generalized quadratic serial rank statistics of the form

$$
Q_{A, B ; f}^{(n) *}=n \mathbf{T}_{\Psi ; f}^{(n)} \mathbf{W}_{\Psi}^{-2} \mathbf{T}_{\Psi ; f}^{(n)}
$$


Actually, we shall show that $Q_{A, B ; f}^{(n) *}$ provides the asymptotically maximin most powerful test for our problem. We first establish an important property that $Q_{A, B ; f}^{(n)^{*}}$, unlike $\mathbf{T}_{\Psi ; f}^{(n)}$, does not depend on the particular fundamental system $\left\{\Psi_{t}^{(i)}\right\}$ adopted.

Proposition 4.1. The quadratic statistic $\mathrm{Q}_{A, \mathrm{~B} ; \mathrm{f}}^{(\mathrm{n}) *}$ does not depend on the fundamental system $\left\{\Psi_{t}^{(i)}\right\}$.

Proof: Let $\left\{\theta_{t}^{(1)}, \ldots, \theta_{t}^{\left(p_{1}+q_{1}\right)}\right\}$ and $\left\{\Psi_{t}^{(1)}, \ldots, \Psi_{t}^{\left(p_{1}+q_{1}\right)}\right\}$ denote two arbitrary fundamental systems for Eq. 19. Then $\left(\theta_{t}^{(1)}, \ldots, \theta_{t}^{\left(p_{1}+q_{1}\right)}=\left(\Psi_{t}^{(1)}, \ldots, \Psi_{t}^{\left(p_{1}+q_{1}\right)}\right) \mathbf{K}^{\prime}, t \in \mathbb{Z}\right.$, where $\mathbf{K}$ is some nonsingular constant matrix of dimension $\left(p_{1}+q_{1}\right)$ $\times\left(p_{1}+q_{1}^{\prime}\right)$. Hence

$$
\mathbf{T}_{\theta ; f .}^{(n)}=\left(\begin{array}{cc}
I & 0 \\
0 & \mathbf{K}
\end{array}\right) \mathbf{T}_{\Psi ; f}^{(n)}, \quad \text { and } \quad W_{\theta}^{2}=\mathbf{K} W_{\Psi}^{2} \mathbf{K}^{\prime}
$$

Thus

$$
\begin{aligned}
\mathbf{T}_{\theta ; f}^{(n)^{\prime}} \mathbf{W}_{\theta}^{-2} \mathbf{T}_{\theta ; f}^{(n)} & \\
& =\mathbf{T}_{\Psi ; f}^{(n)}\left(\begin{array}{cc}
I & 0 \\
0 & \mathbf{K}
\end{array}\right)\left(\begin{array}{cc}
I & 0 \\
0 & \mathbf{K}^{-1} W_{\Psi}^{-2} \mathbf{K}^{\prime-1}
\end{array}\right)\left(\begin{array}{cc}
1 & 0 \\
0 & \mathbf{K}
\end{array}\right) \mathbf{T}_{\Psi ; f}^{(n)} \\
& =\mathbf{T}_{\Psi ; f}^{(n)} \mathbf{W}_{\Psi}^{-2} \mathbf{T}_{\Psi ; f}^{(n)} .
\end{aligned}
$$

Let us illustrate the above proposition by giving an example of a particular formulation of $Q_{A, B}^{(n)}$.

Example 4.1: Denote by $\lambda_{(1)}, \ldots, \lambda_{\left(p_{1}+q_{1}\right)}$ the roots of $A\left(z^{-1}\right) B\left(z^{-1}\right)=0, z \in \mathbb{C}$, and assume that they all are distinct (hence of multiplicity one-also recall that they all lie inside the unit circle). Then a fundamental system of solutions of Eq. 19 is provided by $\left\{\Psi_{t}^{(j)}=\lambda_{j}^{t-(\pi+1)}, t \in \mathbb{Z} ; j=1\right.$, $\left.\ldots, p_{1}+q_{1}\right\} . Q_{A, B}^{(n) *}$ is thus a quadratic form involving the rank autocorrelations of orders 1 through $\pi$ and geometrically weighted sums of rank autocorrelations of orders $\pi+1$ through $n-2$. $W_{\Psi}^{2}$ is of the form $\left(W_{\Psi ; k l}\right)=\left(\left(1-\lambda_{k} \lambda_{l}\right)^{-1}\right)$.

We now give the asymptotic distribution of $Q_{A, B ; f}^{(n) *}$

PROPOSITION 4.2. Under $\mathrm{H}^{(\mathrm{n})}(\mathbf{A}, \mathrm{B} ; \cdot), \mathrm{Q}_{A, \mathrm{~B} ; \mathrm{f}}^{(\mathrm{n}) *}$ is asymptotically $\chi^{2}$ with $\max \left(\mathrm{p}_{1}+\mathrm{q}_{2}, \mathrm{p}_{2}+\mathrm{q}_{1}\right)$ degrees of freedom. Un$\operatorname{der} \mathbf{K}^{(\mathrm{n})}(\mathbf{A}, \mathbf{B} ; \boldsymbol{\gamma}, \boldsymbol{\delta} ; \mathbf{f})$, it is asymptotically noncentral $\chi^{2}$, with the same degrees of freedom and with noncentrality parameter

$$
\lambda_{\mathbf{f}}^{*}(\boldsymbol{\gamma}, \boldsymbol{\delta})=1 / 2\|\mathbf{a}+\mathbf{b}\|^{2} \sigma^{2} \mathrm{I}(\mathbf{f}),
$$

where $\mathrm{a}$ and $\mathrm{b}$ are given by Eq. 6.

Proof: The proof follows straightforwardly from Proposition 4.1. Note that by letting $\mathbf{k}^{\prime}=\left(\left(a_{1}+b_{1}\right), \ldots,\left(a_{\pi}+b_{\pi}\right)\right.$, $\left.k_{1}, \ldots, k_{p_{1}+q_{1}}\right)$, the asymptotic mean of $n^{\mathrm{i} / 2} \mathbf{T}_{\Psi ; f}^{(n)}$ under $K^{(n)}(A, B ; \gamma, \delta ; f)$ takes the form $\mathbf{W}_{\Psi}^{2} \mathbf{k}\left[\sigma^{2} I(f)\right]^{1 / 2}$. On the other hand,

$$
\mathbf{k}^{\prime} \mathbf{W}_{\mathbf{\Psi}}^{2} \mathbf{k}=\|\mathbf{a}+\mathbf{b}\|^{2}
$$

yielding the noncentrality parameter (Eq. 24).

Asymptotically Maximin Most Powerful Test for Testing an ARMA Model Against Unspecified ARMA Alternatives. Let us define an asymptotically maximin most powerful test for $H^{(n)}(\mathbf{A}, \mathbf{B} ; \cdot)$ against an unspecified $\operatorname{ARMA}\left(p_{2}, q_{2}\right)$ alternative as a test that is asymptotically maximin most powerful against any alternative of the form $K^{(n)}\left(\mathbf{A}, \mathbf{B} ; \mathbb{R}^{p_{2}}, \mathbb{R}^{q_{2}} ; f \mid d\right)$ (cf. Eq. 18), $d \in \mathbb{R}^{+}$. We then have the following result.

PROPOSITION 4.3. An asymptotically maximin most powerful test for $\mathrm{H}^{(\mathrm{n})}(\mathrm{A}, \mathrm{B} ; \cdot)$ against an unspecified $\operatorname{ARMA}\left(\mathrm{p}_{2}, \mathrm{q}_{2}\right)$ alternative is provided by

$\psi_{\alpha}^{(\mathrm{n})^{*}}\left(\mathrm{~A}, \mathrm{~B} ; \mathrm{p}_{2}, \mathrm{q}_{2} ; \mathrm{f}\right)=1$

$$
\text { if } Q_{A, B ; f}^{(n) *}>\chi_{\max \left(p_{1}+q_{2}, p_{2}+q_{1}\right) ; 1-\alpha}^{2}
$$

Accordingly, the envelope power function $\beta\left(\alpha, \mathrm{H}^{(\mathrm{n})}(\mathbf{A}\right.$, $\mathbf{B} ; \cdot), \mathrm{K}^{(\mathrm{n})}\left(\mathbf{A}, \mathbf{B} ; \mathbb{R}^{\mathrm{p}_{2}}, \mathbb{R}^{\mathrm{q}_{2}} ; \mathrm{f} \mid \mathrm{d}\right)$ converges, as $\mathrm{n} \rightarrow \infty$, to $1-$ $\mathrm{F}_{\max \left(\mathrm{p}_{1}+\mathrm{q}_{2}, \mathrm{p}_{2}+\mathrm{q}_{1}\right)}\left(\chi_{\max \left(\mathrm{p}_{1}+\mathrm{q}_{2}, \mathrm{p}_{2}+\mathrm{q}_{1}\right) ; 1-\alpha}^{2} ; 1 / 2 \mathrm{~d}^{2} \sigma^{2} \mathrm{I}(\mathrm{f})\right)$, where $\chi_{\mathrm{m} ; 1-\alpha}^{2}$ denotes the $(1-\alpha)$-quantile of the $\chi^{2}$ distribution with $\mathrm{m}$ degrees of freedom and $\mathrm{F}_{\mathrm{m}}(\cdot ; \lambda)$, the distribution function of the noncentral $\chi^{2}$ with $\mathrm{m}$ degrees of freedom and noncentrality parameter $\lambda$.

COROLlaRY 4.4. The rank portmanteau statistic n $\Sigma_{\mathrm{i}=1}^{\mathrm{p}}$ $\left(\mathrm{r}_{\mathrm{i}: \mathrm{f}}^{(\mathrm{n})}\right)^{2}$ [cf. Hallin et al. (2)] is asymptotically maximin most powerful against unspecified $A R M A\left(\mathrm{p}_{2}, \mathrm{q}_{2}\right)$ alternatives if and only if $\mathrm{p}_{1}=\mathrm{q}_{1}=0$ and $\mathrm{p}=\max \left(\mathrm{p}_{2}, \mathrm{q}_{2}\right)$ (i.e., for testing randomness against serial dependence of order $\mathrm{p})$.

Proof: We know from Section 3 that $\mathbf{T}_{\Psi ; f}^{(n)}$ is asymptotically sufficient for testing $H^{(n)}(\mathbf{A}, \mathbf{B} ; \cdot)$ against Eq. 18. $n^{1 / 2} \mathbf{W}_{\Psi}^{-1} \mathbf{T}_{\Psi ; f}^{(n)}$ is thus also asymptotically sufficient, since $\mathbf{W}_{\Psi}^{2}$ is a full-rank covariance matrix.

Under $K^{(n)}(A, B: \gamma, \delta ; f)$, the asymptotic distribution of $n^{1 / 2} \mathbf{W}_{\Psi}^{-1} \mathbf{T}_{\Psi ; f}^{(n)}$ is normal, with mean $\mu(\gamma, \delta)=\mathbf{W}_{\Psi} \mathbf{k}\left[\sigma^{2} I(f)\right]^{1 / 2}$ and identity covariance matrix.

Let us show that when $(\boldsymbol{\gamma}, \boldsymbol{\delta})$ describes $\mathbb{R}^{p_{2}} \times \mathbb{R}^{q_{2}}, \mathbf{k}$ and thus $\boldsymbol{\mu}(\boldsymbol{\gamma}, \boldsymbol{\delta})$ describes $\mathbb{R}^{\max \left(p_{1}+q_{2}, p_{2}+q_{1}\right)}$. Any value of $\left(a_{1}\right.$, ..., $\left.a_{p_{2}-p_{1}}\right) \in \mathbb{R}^{p_{2}-p_{1}}$ can be reached by choosing appropriate values of $\gamma_{1}, \ldots, \gamma_{q_{2}-q_{1}}$, and $a_{t}, t \geq p_{2}-p_{1}+1$ can be made equal to any solution of $A(L) \Psi_{t}=0$ by adjusting the remaining $p_{1}$ components $\gamma_{p_{2}-p_{1}+1}, \ldots, \gamma_{p_{2}}$ of $\boldsymbol{\gamma}$. A similar result holds for $b$ and $\delta$. Furthermore, any solution of $A(L) B(L) \Psi_{t}=0, t \geq \pi+1$, can be decomposed into a solution $a_{t}$ of $A(L) \Psi_{t}=0$ plus a solution $b_{t}$ of $B(L) \Psi_{t}=0$. This follows indeed from the fact that $A(z)$ and $B(z)$ have no common root; a fundamental system for $A(L) B(L) \Psi_{t}=0$ can thus be formed by juxtaposing a fundamental system $\left\{\Psi_{t}^{(1)}\right.$, $\left.\Psi_{t}^{\left(p_{1}\right)}\right\}$ of $A(L) \Psi_{t}=0$ with a fundamental system $\left\{\Psi^{\left(p_{1}+1\right)}\right.$, $\left.\ldots, \Psi^{\left(p_{1}+q_{1}\right)}\right\}$ of $B(L) \Psi_{t}=0$.

Now, under the condition $(\boldsymbol{\gamma}, \boldsymbol{\delta}) \in \mathscr{D}(d)$, i.e., $\|\mathbf{a}+\mathbf{b}\|^{2} \geq$ $d^{2}$, the values of $\mu(\gamma, \delta)$ are restricted to those satisfying $\mu^{\prime}(\gamma, \delta) \mu\left(\gamma, \delta^{\prime}\right)=\mathbf{k}^{\prime} \mathbf{W}_{\Psi}^{2} \mathbf{k} \sigma^{2} I(f) \geq d^{2} \sigma^{2} I(f)$ (cf. Proposition 4.2). Thus the family of asymptotic distributions of $n^{1 / 2} W_{\Psi}^{-1}$ $T_{\Psi ; f}^{(n)}$ under $K^{(n)}\left(A, B ; \mathbb{R}^{p_{2}}, \mathbb{R}^{q_{2}} ; f \mid d\right)$ is the set of all normal distributions with mean $\boldsymbol{\mu}$ and identity covariance matrix such that $\mu^{\prime} \mu \geq d^{2} \sigma^{2} I(f)$, whereas under $H^{(n)}(\mathbf{A}, \mathbf{B} ; \cdot)$ the asymptotic distribution of $n \mathbf{W}_{\Psi}^{-1} T_{\Psi ; f}^{(n)}$ is normal with mean 0 and identity covariance matrix.

It then follows from an invariance argument and the HuntStein theorem that the maximin most powerful test for this problem is based on the test statistic $n \mathbf{T}_{\Psi ; f}^{(n)} \mathbf{W}_{\Psi}^{-2} \mathbf{T}_{\Psi ; f}^{(n)}-$ which is precisely $Q_{A, B ; f}^{(n)}$. Because of the asymptotic sufficiency of $\mathbf{T}_{\Psi ; f}^{(n)}$, this test is also maximin most powerful for $H^{(n)}(\mathbf{A}, \mathbf{B} ; \cdot)$ against any $K^{(n)}\left(\mathbf{A}, \mathbf{B} ; \mathbb{R}^{p_{2}}, \mathbb{R}^{q_{2}} ; f \mid d\right), d \in \mathbb{R}^{+}$.

Corollary 4.4 gives the particular form of the asymptotically maximin most powerful statistic $Q_{A, B ; f}^{(n)}$ in the case where $p_{1}=q_{1}=0$ and $\pi=\max \left(p_{2}, q_{2}\right)$. This is the only case where $Q_{A, B ; f}^{(n) *}$ turns out to be a quadratic serial rank statistic in the sense of Hallin et al. (2).

Remark 4.1: Denoting by $Q_{f}^{(n)}=n \Sigma_{i=1}^{\pi}\left(r_{i ; f}^{(n)}\right)^{2}$ the rank portmanteau statistic of order $\pi=\max \left(p_{2}-p_{1}, q_{2}-q_{1}\right)$, maximin optimal quadratic statistics $Q_{A, B ; f}^{(n)^{*}}$ can be decomposed into $Q_{A, B ; f}^{(n)^{*}}=Q_{f}^{(n)}+Q_{A, B ; f}^{(n)}$. Because $Q_{f}^{(n)}$ depends only on $r_{1 ; f}^{(n)} \ldots, r_{\pi ; f}^{(n)}$ whereas $Q_{A, B ; f}^{(n)}$ depends only on $r_{\pi+1 ; f}^{(n)}, r_{\pi+2 ; f}^{(n)}$, $\ldots, Q_{f}^{(n)}$ and $Q_{A, B ; f}^{(n)}$ are asymptotically independent $\chi^{2}$-distributed statistics [under $H^{(n)}(\mathbf{A}, \mathbf{B} ; \cdot)$ ]. $Q f^{(n)}$ accounts for those models in the alternative that differ from the tested model because of the existence of higher order terms (autoregressive terms of orders larger than $p_{1}$ and/or moving average terms of orders larger than $q_{1}$ ). It contributes for $\max \left(p_{2}-p_{1}, q_{2}-q_{1}\right)$ degrees of freedom, the maximal number of such possible higher order terms. $Q_{A, B ; f}^{(n)}$, which has the form of a weighted rank portmanteau statistic accounts for those models in the alternative that differ from the tested model because their autoregressive coefficients of orders 1 
through $p_{1}$ and moving average coefficients of orders 1 through $q_{1}$ differ from the tested ones (i.e., the $A_{i}$ and $B_{i}$ values). It contributes for $\left(p_{1}+q_{1}\right)$ degrees of freedom, one for each tested coefficient $A_{1}, \ldots, A_{p_{1}}, B_{1}, \ldots, B_{q_{1}}$. The consideration of this decomposition in the case when $H^{(n)}(\mathbf{A}$, B; $\cdot)$ has been rejected may provide a useful insight into the reasons why rejection occurred and thus suggest alternative model specifications.

Remark 4.2: The noncentrality parameter $\lambda_{f}^{*}(\boldsymbol{\gamma}, \delta)$ of the asymptotic distribution of $Q_{A, B ; f}^{(n)^{*}}$ under alternatives of the form $K^{(n)}(A, B ; \gamma, \delta ; f)$ also can be decomposed into $\frac{1}{2} \Sigma_{i=1}^{\pi}$ $\left(a_{i}+b_{i}\right)^{2} \sigma^{2} I(f)$ (contribution of the "unweighted part" $\left.Q_{f}^{(n)}\right)$ and $1 / 2 \Sigma_{i=\pi+1}^{\infty}\left(a_{i}+b_{i}\right)^{2} \sigma^{2} I(f)$ (contribution of the "weighted part" $Q_{A, B ; f}^{(n)}$. A study of the relation between these two quantities and the coefficients of the tested model (Eq. 2) provides an interesting insight into the importance of the respective contributions of the "weighted" and "unweighted" parts to the asymptotic power of Eq. 25. As a general rule, the contribution of the unweighted part does not depend on $A(L)$ and $B(L)$, whereas the contribution of the weighted part is an increasing function of the "closeness to the unit circle" of the roots of $A(z) B(z)=0, z \in \mathbb{C}$. To show this, let us consider a simple example.

Example 4.2: Consider the problem of testing the $A R(1)$ model $X_{t}-\rho X_{t-1}=\varepsilon_{t}, t \in \mathbb{Z}$, when $|\rho|<1$, against ARMA $(2,1)$ alternatives. Here $\pi=1$, and

$$
a_{i}=\left\{\begin{array}{lll}
\gamma_{1} & \text { if } & i=1 \\
\gamma_{1} \rho^{i-1}+\gamma_{2} \rho^{i-2} & \text { if } & i \geq 2
\end{array}\right.
$$

and

$$
b_{i}=\left\{\begin{array}{lll}
\delta_{1} & \text { if } & i=1 \\
0 & \text { if } & i \geq 2
\end{array}\right.
$$

if an alternative of the form $K^{(n)}\left(\mathbf{A}, \mathbf{B} ; \boldsymbol{\gamma}=\left(\gamma_{1}, \gamma_{2}\right), \delta=\left(\delta_{1}\right.\right.$, $0) ; f$ ) is considered. The noncentrality parameter (Eq. 24) accordingly decomposes into $\left(\gamma_{1}+\delta_{1}\right)^{2}$ (contribution of the unweighted $Q_{f^{(n)}}$, which does not depend on $\rho$, and $\Sigma_{i=2}^{\infty}$ $\left(\gamma_{1} \rho^{i-1}+\gamma_{2} \rho^{i}\right)^{2}=\left(\gamma_{1} \rho+\gamma_{2}\right)^{2}\left(1 /\left(1-\rho^{2}\right)\right)($ contribution of the weighted $\left.Q_{A, B ; f}^{(n)}\right)$. Clearly, this latter contribution is approximately $\gamma_{2}^{2}$ if $|\rho| \simeq 0$-and the optimal test (Eq. 25) is then approximately equivalent to the test based on an unweighted rank portmanteau statistic of order two; if $|\rho| \simeq 1$, then the contribution of $Q_{A, B ; f}^{(n)}$ can be arbitrarily large. The absence of such a weighted part in the classical portmanteau statistic could possibly be responsible for the somewhat disappointing performance of the usual Box-Pierce test.

\section{Section 5. Exact and Approximate Residuals}

All the results in the sections above are based on the ranks $R_{t}^{(n)}$ of the exact residuals

$$
Z_{t}=[A(L) / B(L)] X_{t}^{(n)}, \quad t=1, \ldots, n .
$$

Clearly, even in the case of purely autoregressive processes $\left(q_{1}=0\right)$, these exact residuals cannot be all computed from the observations. Replacing them with the approximate residuals [obtained by putting $X_{t}^{(n)}=0, t \leq 0$ in Eq. 26] does not affect any of the asymptotic results given here [cf. Hallin and Puri (5)].

The research of M.L.P. was supported by Office of Naval Research Contract N00014-85-K-0648.

1. Hallin, M., Ingenbleek, J.-F. \& Puri, M. L. (1985) Ann. Stat. 13, 1156-1181.

2. Hallin, M., Ingenbleek, J.-F. \& Puri, M. L. (1985) C.R. Seances Acad. Sci. Ser. A 301, 935-938.

3. Box, G. E. P. \& Pierce, D. A. (1970) J. Am. Stat. Assoc. 65, 1509-1526.

4. Hájek, J. \& Šidák, Z. (1967) Theory of Rank Tests (Academic, New York).

5. Hallin, M. \& Puri, M. L. (1988) Ann. Stat., in press.

6. LeCam, L. (1960) Univ. Calif. Publ. Stat. 3, $27-98$.

7. Anderson, T. W. (1971) The Statistical Analysis of Time Series (Wiley, New York).

8. Hallin, M., Ingenbleek, J.-F. \& Puri, M. L. (1987) J. Time Ser. Anal. 8, 409-424. 